\title{
Giant Hydronephrosis - A Late Diagnosis of Ureteropelvic Junction Obstruction
}

\author{
Luis Sepulveda $^{\mathrm{a}, \mathrm{b}}$, Filipe Rodrigues ${ }^{\mathrm{a}}$
}

\begin{abstract}
Ureteropelvic junction obstruction is the most common cause of neonatal hydronephrosis, with an overall incidence of 1:1,500. Since the widespread use of ultrasound in pregnancy, most cases are discovered early in life; however, if the diagnosis isn't suspected in the newborn and the patient remains asymptomatic, the hydronephrosis can progress over time and reach disproportional dimensions. We report a case of giant hydronephrosis in a 27 -yearold man presented with severe sepsis secondary to pneumonia and incidental finding of abdominal mass. The current literature is also reviewed.
\end{abstract}

Keywords: Giant hydronephrosis; Ureteropelvic junction obstruction; Adult

\section{Introduction}

The ureteropelvic junction (UPJ) obstruction is defined as an impairment of urine flow from the renal pelvis into the proximal ureter, leading to progressive dilatation of the collecting system and potential renal injury [1]. With an overall incidence of $1: 1,500$, it is the most common cause of neonatal hydronephrosis $[1,2]$. It predominantly affects male newborns (2:1), is bilateral in 10 to $40 \%$ of the cases and may be associated with other anomalies like vesico-ureteral reflux, renal dysplasia or agenesis [1,2].

UPJ obstruction can be intrinsic, due to excessive connective tissue and decrease smooth muscle content in the

Manuscript accepted for publication May 13, 2013

\footnotetext{
${ }^{a}$ Urology Department of Centro Hospitalar de Tras-os-Montes e Alto Douro (Chtmad), Portugal

${ }^{b}$ Corresponding author: Luis Sepulveda, Urology Department of Centro Hospitalar de Tras-os-Montes e Alto Douro, Avenida da Noruega 5000-508 Vila Real, Portugal. Email: LuisSepulveda.Uro@gmail.com
}

doi: http://dx.doi.org/10.4021/wjnu95w ureteral wall, or extrinsic, probably because of an anomalous renal vasculature, leading to an aberrant, accessory or earlybranching lower pole vessel. However it is still difficult to define if the presence of an aberrant vessel is the real cause of obstruction or just a co-variable of an intrinsic obstruction [2].

The definition of giant hydronephrosis has been given by Stirling in 1939, as the presence of more than $1,000 \mathrm{~mL}$ or $1.6 \%$ of body weight of fluid in the collecting system [3, 4]. With the increasing use of ultrasound in the prenatal evaluation in the mid 80's, nowadays cases of giant hydronephrosis due to UPJ obstruction are uncommonly diagnosed in adults [5].

We describe a man with incidental finding of severe right renal injury due to chronic ureteropelvic junction obstruction and subsequent formation of giant hydronephrosis, despite absence of symptoms or relevant urologic history.

\section{Case Report}

A 27-year-old man, dumb, deaf and with mild mental retardation, presents in the Emergency Room (ER) with sepsis secondary to community-acquired pneumonia. During assessment in the ER was detected a large painless abdominal

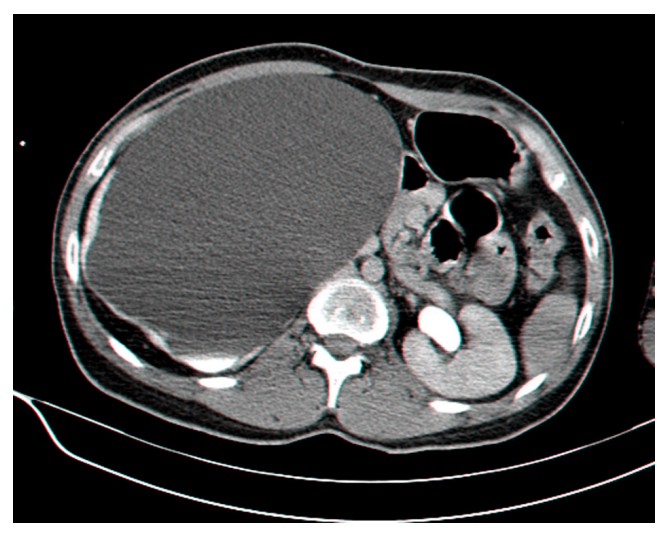

Figure 1. Abdominal CT scan showing giant right hydronephrosis with pressure effect over the bowels and thinning of renal parenchyma. 


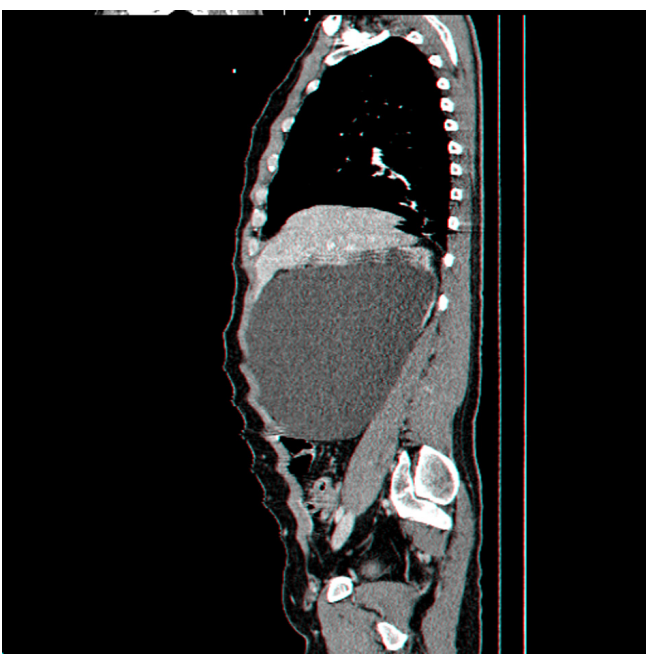

Figure 2. Sagital reconstruction CT scan showing giant right hydronephrosis occupying most of the abdominal cavity.

mass, localized in the right upper quadrant and extending into the pelvis. He had no prior history of colic, urolithiasis, or infection. Computed tomography revealed severe right hydronephrosis and thinning of renal parenchyma (Fig. 1, 2) due to probable congenital UPJ obstruction, with compression of adjacent viscera and lateralization of the great vessels (Fig. 3). The left kidney was normal. Blood analysis presented a normal renal function.

Due to significant compression of intra-abdominal structures and limitation of diaphragmatic excursion, the patient was first submitted to percutaneous nephrostomy and later, when suitable for anesthesia, to ureteral retrograde stenting. The initial drainage following nephrostomy was over two liters of clear urine.

Currently, the patient is schedule for nuclear renography to evaluate the differential renal function and probable right nephrectomy. The patient and her mother were unaware of prior urologic history and denied complications or abnormal exam results during antenatal period. Nonetheless, according to the mother statement, there was an absence of close surveillance with US during prenatal period.

\section{Discussion}

Nowadays most cases of UPJ obstruction are suspected during pregnancy due to the recent widespread use of ultrasonography in the antenatal period, especially after the 28th week when it is most accurate to study the urogenital tract $[1,5]$. After labor, in case of suspicion of UPJ obstruction, is highly recommended close surveillance of the newborn by clinical examination, US and renography with MAG3. Detection of impairment or decrease in split renal function, increase in the antero-posterior diameter, recurrent renal infection and flank pain are the most common indications for

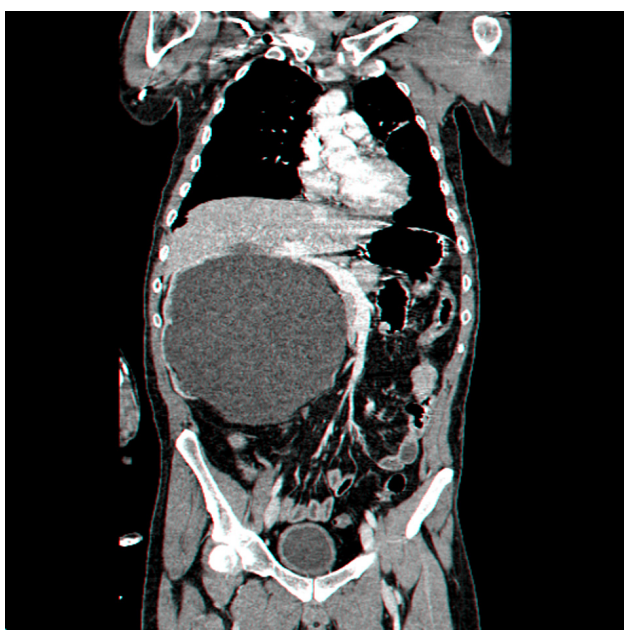

Figure 3. Coronal reconstruction CT scan showing giant right hydronephrosis with pressure effect over the bowels and liv$\mathrm{er}$, associated with lateralization of the great vessels.

surgical treatment $[1,5]$.

Despite most patients being diagnosed in the early years, there are still cases of late diagnosis, including in adulthood, usually associated to an asymptomatic evolution [6]. The delay in detection of UPJ obstruction can lead to progressive and severe hydronephrosis and loss of function of the affected kidney at the time of diagnosis $[1,6]$.

The case presented documents an incidental finding of severe renal injury secondary to congenital UPJ obstruction in an adult patient with no urologic symptoms or prior known antenatal or childhood urologic history. The highlights of this case are the asymptomatic progression of an ureteropelvic junction obstruction, leading to severe injury of renal parenchyma and giant dilatation of the collecting system. Despite the presentation as a meaningful and easily palpable abdominal mass, neither the patient nor the family seemed to be particularly concerned with its nature and evolution.

\section{References}

1. S. Tekgul, H. Riedmiller, H.S.Dogan, et al.; members of the European Association of Urology (EAU) Guidelines Office. Guidelines on Paediatric Urology, In: EAU Guidelines, edition presented at the 25th EAU Annual Congress, Barcelona 2010. ISBN 978-90-79754-70-0.

2. Hiep Thieu Nguyen, Bulent Onal. Chapter 2: Pediatric Urology. In: AUA Educational Review Manual in Urology; 3rd Edition 2011; 2:25-56.ISBN 978-0-9846361-05.

3. Chiang PH, Chen MT, Chou YH, Chiang CP, Huang $\mathrm{CH}$, Chien $\mathrm{CH}$. Giant hydronephrosis: report of 4 cases with review of the literature. J Formos Med Assoc. 1990;89(9):811-817. 
4. Tazi MF, Riyach O, Ahallal Y, Mellas S, Khallouk A, El Fassi MJ, Farih MH. Giant Urinary Bladder and Bilateral Giant Hydronephrosis due to Bladder Neck Obstruction: One Case Report and Literature Review. Case Rep Urol. 2012;2012:817519.

5. Molina CA, Facincani I, Muglia VF, Araujo WM, Cas- sini MF, Tucci S, Jr. Postnatal evaluation of intrauterine hydronephrosis due to ureteropelvic junction obstruction. Acta Cir Bras. 2013;28(Suppl 1):33-36.

6. Hellenthal NJ, Thomas SA, Low RK. Rapid onset renal deterioration in an adult with silent ureteropelvic junction obstruction. Indian J Urol. 2009;25(1):132-133. 